Bundesgesundheitsbl 2020 63:698-707 https://doi.org/10.1007/s00103-020-03156-1 Online publiziert: 25 . Mai 2020

(c) Der/die Autor(en) 2020

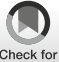

Daniel Tolks ${ }^{1,2} \cdot$ Claudia Lampert $^{3} \cdot$ Kevin Dadaczynski $^{1,4} \cdot$ Eveline Maslon $^{5} \cdot$ Peter Paulus ${ }^{1} \cdot$ Michael Sailer $^{6}$

'Zentrum für Angewandte Gesundheitswissenschaften, Leuphana Universität Lüneburg, Lüneburg, Deutschland

${ }^{2}$ Institut für Didaktik und Ausbildungsforschung in der Medizin, Klinikum der Universität München, Ludwig-Maximilians-Universität München, München, Deutschland

${ }^{3}$ Leibniz-Institut für Medienforschung, Hamburg, Deutschland

${ }^{4}$ Fachbereich Pflege und Gesundheit, Hochschule Fulda, Fulda, Deutschland

${ }^{5}$ Bundeszentrale für gesundheitliche Aufklärung, Köln, Deutschland

${ }^{6}$ Lehrstuhl für Empirische Pädagogik und Pädagogische Psychologie, Ludwig-Maximilians-Universität München, München, Deutschland

\title{
Spielerische Ansätze in Prävention und Gesundheitsförderung: Serious Games und Gamification
}

dungen in der Prävention und Gesundheitsförderung. Ausgeklammert werden dabei die möglichen negativen Auswirkungen einer exzessiven Computerspielenutzung. Die beiden spielerischen Ansätze Serious Games und Gamification, die das größte Potenzial für den Einsatz im Bereich der Prävention und Gesundheitsförderung zeigen, sollen beschrieben werden.

\section{Serious Games}

Der Einsatz von Spielen zu ernsthaften Zwecken reicht zurück bis zum Beginn der Menschheitsgeschichte und ist heute noch integraler Bestandteil der menschlichen Kultur [2]. Der Begriff „Serious Games" selbst wurde erstmals 1970 von Clark C. Abt verwendet [3]. Sawyer und Kollegen beschreiben Serious Games als „any computerized game whose chief mission is not entertainment and all entertainment games which can be reapplied to a different mission other than entertainment" [4]. Bei den Serious Games handelt es sich vornehmlich um digitale Anwendungen mit spielerischen und didaktischen Anteilen [5], obwohl in einigen Definitionen auch nichtdigitale
Spiele unter diese Kategorie subsumiert werden [6].

Das zentrale Abgrenzungsmerkmal gegenüber entertainmentorientierten Spielen ist ein explizit formuliertes Bildungsziel [7]. Dieses bzw. der pädagogische Inhalt muss sich dem Unterhaltungsfaktor unterordnen, d.h., das Unterhaltungserleben des Spielenden sollte im Vordergrund stehen. Die Ziele der Implementierung von Lernszenarien in Spielen sind der Wissenszuwachs, die Vertiefung von Kompetenzen und die Erzeugung einer intendierten Verhaltensänderung [5]. Dabei kommen lernund motivationsförderliche Aspekte von Spielen, wie z. B. Immersion (das „Eintauchen " in virtuelle Spielwelten), Storytelling (Erzählen einer Geschichte), eine differenzierte und visuell ansprechende Spielumgebung sowie das Erzeugen eines Flowerlebnisses (die vollkommene Balance zwischen Anforderungen und Bewältigung), zur Anwendung [8]. Der Immersionsfaktor sowie das Flowerleben unterstützen den Lernprozess [8].

Serious Games bieten ein großes Potenzial, nicht nur die Motivation, sondern auch den Lernerfolg - über die kognitiven Lernziele hinaus - auf affektiver und psychomotorischer Ebene zu steigern [9]. 


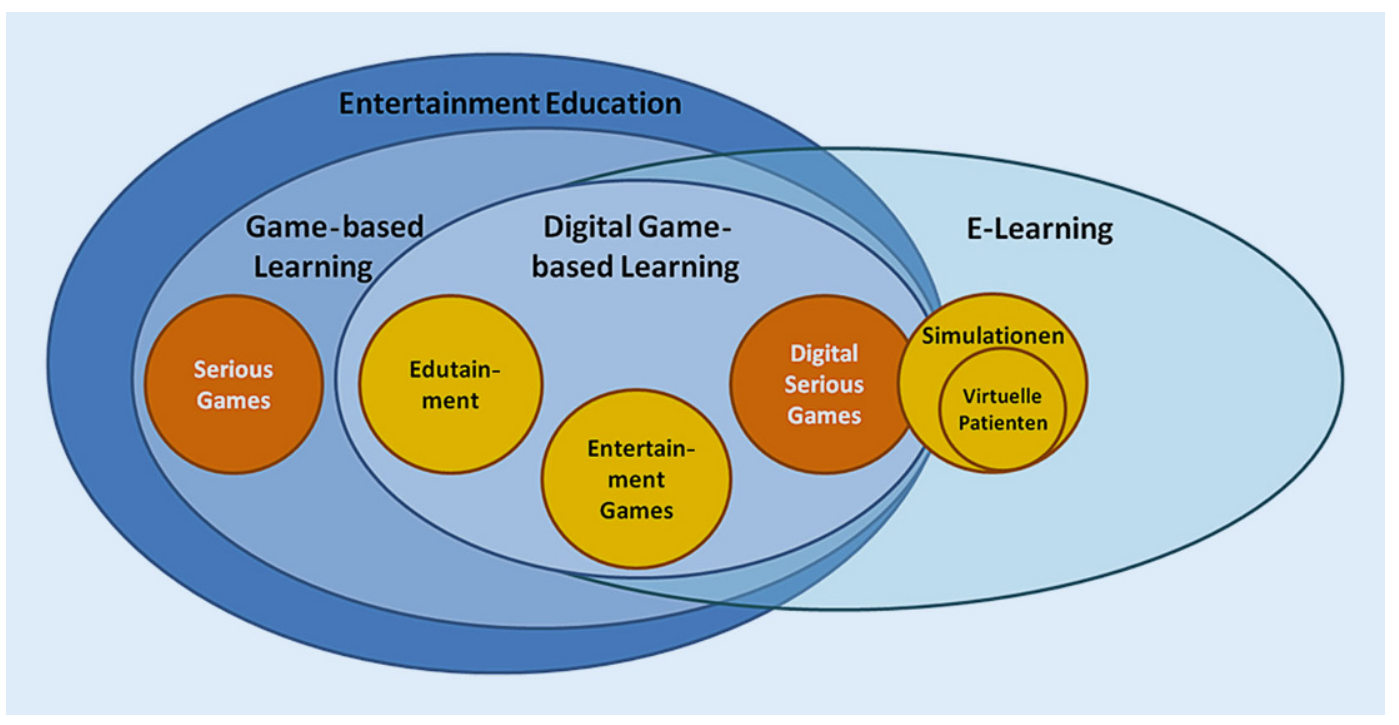

Abb. $1<$ Abgrenzung von Serious Games zu anderen Lehr- und Lernmethoden [7]. (Abbildung mit freundlicherGenehmigung (c) Beltz Juventa Verlag)

Folgende Kriterien sind für die Einstufung von spielerischen Anwendungen als Serious Game notwendig [7]:

- Die Intention des Spieles ist die Vermittlung von Inhalten, die nicht primär der Unterhaltung dienen.

- Das Spiel muss einen pädagogischen Inhalt besitzen.

- Der pädagogische Inhalt muss sich dem Unterhaltungsfaktor unterordnen.

- Das Spiel muss auf mindestens ein Lernziel abzielen.

Wie in - Abb. 1 dargestellt, existieren neben dem Serious-Games-Ansatz zahlreiche weitere digital gestützte Lehrund Lernmethoden, die aufgrund einer fehlenden einheitlichen Begriffsverwendung zu einer heterogenen Forschungslage geführt haben $[7,10]$. Eine ausführliche Beschreibung der unterschiedlichen Begriffe ist in dem Beitrag von Tolks und Lampert zu finden [7]. Begriffe wie "Games for Impact/Change“ (Spiel mit sozialen Auswirkungen/für Veränderung) oder "Game-based Learning“ (spielbasiertes Lernen) werden oft synonym genutzt. Als besonders relevant erweist sich hierbei auch der Dachbegriff der „Entertainment Education“, der sich auf die Nutzung von unterhaltungsorientierten Ansätzen für Bildungsanliegen bezieht [11]. Dem steht der umfassende Begriff „E-Learning“ gegenüber, der alle Informations- und Kommunikationstechniken subsumiert, mit deren
Unterstützung ein Lernprozess gefördert werden kann.

\section{Gamification}

Anfang der 2000er-Jahre konnten im Rahmen einer Studie einzelne Spieldesignelemente identifiziert werden, die einen direkten Einfluss auf die Motivation und Leistung der Nutzenden zeigten [12]. Diese Studie legte den Grundstein für das Konzept von Gamification, das nicht nur im Business- und Marketingsektor, sondern auch in der Wissenschaft an Bedeutung gewonnen hat [13].

Die am häufigsten verwendete Definition dieses Konzepts lautet: „,The use of game-elements and game-design techniques in non-game contexts "[14]. Gamification umschreibt die Idee, Spieldesignelemente in spielfremden Kontexten zu verwenden, um die Motivation, die Leistung und das Engagement der Nutzenden zu erhöhen [15].

Die Forschungsfelder Gamification und Serious Games haben überlappende Forschungsliteratur und greifen beide auf die gleichen Werkzeuge, nämlich Spieldesignelemente, zurück [16]. Darüber hinaus wird bei beiden Ansätzen ein Mehrwert angestrebt, der über die bloße Unterhaltung hinausgeht. Trotz dieser Gemeinsamkeiten, sind die beiden Konzepte voneinander abzugrenzen: Während Serious Games das Design von vollwertigen Spielen implizieren, steht bei Gamification die Anreicherung eines bereits existierenden Prozesses oder Produktes im Fokus mit dem Ziel, eine spielerische Version des Existierenden zu erhalten [16, 17].

Werbach und Hunter identifizierten insgesamt 15 Spieldesignelemente, die einen Einfluss auf das Nutzungsverhalten haben [15]. Dazu gehören beispielsweise Punkte, Bestenlisten, Level-ups, Quests (Aufgaben oder Missionen) und Badges (virtuelle Auszeichnungen). Durch die Nutzung dieser Elemente sollen die Motivation und das Engagement der Nutzenden durch eine spielähnliche Erfahrung gefördert werden [18].

Die motivationale Wirkung der Spieldesignelemente lässt sich auf unterschiedliche Weise erklären. So stellt Sailer den Bezug zwischen verschiedenen Gamificationelementen und der Selbstbestimmungstheorie von Ryan und Deci her [19, 20]. Diese definiert als Motivationstheorie 3 universelle psychologische Grundbedürfnisse, die das menschliche Handeln bestimmen: (1) Kompetenz, (2) Autonomie sowie (3) soziale Eingebundenheit. Werden ein oder mehrere dieser Bedürfnisse (u. a. durch Gamificationelemente) angesprochen, so hat dies positive Effekte auf das Verhalten sowie seine Determinanten (vgl. - Tab. 1). Die am häufigsten genutzten Spieldesignelemente sind Punkte, Auszeichnungen und Bestenlisten [15]. Eine Studie von $\mathrm{Xi}$ und Hamari konnte nachweisen, dass der Einsatz von Auszeichnungen so- 
gar Einfluss auf alle 3 psychologischen Grundbedürfnisse zeigt [21].

\section{Serious Games zur Prävention und Gesundheitsförderung}

In den letzten Jahren hat unter der Bezeichnung „Serious Games for Health“ ein deutlicher Entwicklungsschub eingesetzt [22, 23]. Einige Meilensteine sind in den USA die Gründung der Gamesfor-Health-Initiative im Jahr 2004 und des Games for Health Journal 2012 durch Tom Baranowski, in Deutschland sind es die Serious Games Conference auf der Messe CeBIT, im Jahre 2016 das Buch Gesundheit spielend fördern sowie 2018 das Schwerpunktheft „Digitale Gesundheitskommunikation durch spielerische Ansätze" in der Zeitschrift Prävention und Gesundheitsförderung [1, 2, 24].

Bei den Serious Games for Health lassen sich grundsätzlich 3 Spieleformen unterscheiden $[5,25]$ :

- Computerspiele, die gesundheitsrelevante Informationen, Einstellungen und Verhaltensänderung im Bereich Prävention und Gesundheitsförderung vermitteln,

- Computerspiele, die zu therapeutischen Zwecken bei psychischen und physischen Erkrankungen eingesetzt werden,

- Serious Games, die in der medizinischen Aus-, Weiter- und Fortbildung zum Einsatz kommen.

Lu und Kharrazi konnten im Jahr 2018 in ihrer Übersichtsarbeit 1743 Games for Health aus 23 Ländern identifizieren [26]. Der größte Anteil stammte hierbei aus den USA (67\%), während Deutschland mit lediglich 2,8\% vertreten war. Mit Blick auf die Zielgruppen ist die Mehrzahl der Games for Health für die allgemeine Bevölkerung entwickelt worden (84\%). Demgegenüber wurden etwa $9 \%$ der Spiele für professionelle Akteure im Gesundheitswesen und $7 \%$ für Patienten mit spezifischen Erkrankungen entwickelt. Inhaltlich handelte es sich bei einem Großteil der Spiele nur um simple Puzzlespiele (43\%). Etwa $20 \%$ der Anwendungen verfügten über eine Art Erzählstruktur. Überdies waren $75 \%$ mit einer Gesamtspieldauer von einer Stunde recht

Bundesgesundheitsbl 2020 -63:698-707 https://doi.org/10.1007/s00103-020-03156-1 (c) Der/die Autor(en) 2020

D. Tolks · C. Lampert · K. Dadaczynski · E. Maslon · P. Paulus · M. Sailer

\section{Spielerische Ansätze in Prävention und Gesundheitsförderung: Serious Games und Gamification}

\section{Zusammenfassung}

Digitale Spieleanwendungen können im Bereich Prävention und Gesundheitsförderung eingesetzt werden, um etwa gesundheitsrelevante Informationen zu vermitteln oder Verhaltensänderungen zu evozieren. Dabei sind die beiden relevantesten spielerischen Ansätze Serious Games (Spiele mit ernsthaftem Hintergrund) und Gamification (Anwendung spieltypischer Elemente in spielfremden Kontexten). Der Begriff Serious Games umschreibt Spiele, die eingesetzt werden, um ernsthafte Inhalte, wie zum Beispiel gesundheitliche Themen, zu vermitteln. Bei Gamification werden Spieldesignelemente, wie beispielsweise Punkte, Bestenlisten, Auszeichnungen, Profilgestaltung und Teamevents, eingesetzt, um eine Motivations- und Leistungssteigerung etwa in Lernumgebungen zu bewirken. Der vorliegende narrative Beitrag beleuchtet die Wirkungsweise und Studienlage sowie die Vor- und Nachteile spielerischer Anwendungen in der Prävention und Gesundheitsförderung und führt einige dieser Anwendungen exemplarisch auf. Serious Games und Gamification zeigen in der Prävention und Gesundheitsförderung ein großes Potenzial. Insbesondere bei schwer erreichbaren und desinteressierten Zielgruppen kann ein direkter Bezug zu deren Lebenswelt hergestellt werden. Grundlagen für den wirkungsvollen Einsatz spielerischer Ansätze in der Arbeit und Ausbildung von Gesundheitsberufen sind die bereits hohe Vertrautheit mit dem Medium Spiel sowie die direkte Adressierung der psychologischen Grundbedürfnisse zur Steigerung der Motivation.

Schlüsselwörter

Spiel-Design-Elemente · Game-based learning · Wellbeing Game · Selbstbestimmungstheorie - Onya

\section{Game-based approaches to prevention and health promotion: serious games and gamification}

\section{Abstract}

Digital game-based approaches can be used in the field of prevention and health promotion, for example to promote healthrelated information or to foster health behavior change. The two most relevant game approaches are "serious games" (games with a serious background) and "gamification" (the application of gametypical elements in nongame contexts). The term serious games is used to describe games that are used to convey serious content, such as health issues. Gamification uses gamedesign elements such as points, leaderboards, awards, profile design, and team events to increase motivation and performance, for example in learning environments. This narrative article examines the mode of action and study situation as well as the advantages and disadvantages of playful applications in prevention and health promotion and exemplifies some of these applications. Serious games and gamification show great potential in prevention and health promotion. Especially in the case of target groups that are difficult to reach and disinterested, a direct connection to their living environment can be established. The foundations for the effective use of playful approaches in the work and training of health professionals are their already high level of familiarity with the medium of games and that games directly address the basic psychological needs to increase motivation.

\section{Keywords}

Game-Design-Elements · Game-based learning $\cdot$ Wellbeing game $\cdot$ Self-Determination Theory · Onya kurz. Hinsichtlich möglicher Wirkungen ließen sich in einer Metaanalyse von DeSmet et al. unter Einbezug von 54 Studien die größten Effekte beim Wissenserwerb feststellen, während Connolly und Kollegen in ihrer Literaturübersicht zu- dem empirische Belege für die positiven Auswirkungen auf kognitive, wahrnehmungsbezogene, verhaltensbezogene, affektive und motivationale Aspekte fanden [27, 28]. 
Tab. 1 Wirkung von Spieldesignelementen auf die psychologischen Grundbedürfnisse. (Mod. n. Sailer [19])

\begin{tabular}{|c|c|}
\hline $\begin{array}{l}\text { Psychologisches } \\
\text { Bedürfnis }\end{array}$ & Spieldesignelement \\
\hline \multirow{6}{*}{$\begin{array}{l}\text { Kompetenz- } \\
\text { erleben }\end{array}$} & Punkte \\
\hline & Leistungsanzeige \\
\hline & $\begin{array}{l}\text { Auszeichnungen (Bad- } \\
\text { ges) }\end{array}$ \\
\hline & Bestenliste \\
\hline & Levelsystem \\
\hline & Aufgaben (Quests) \\
\hline \multirow[t]{3}{*}{ Autonomie } & Profilgestaltung \\
\hline & Avatare \\
\hline & $\begin{array}{l}\text { Freie Gestaltung der } \\
\text { Aktivitäten }\end{array}$ \\
\hline \multirow{5}{*}{$\begin{array}{l}\text { Soziale } \\
\text { Eingebundenheit }\end{array}$} & Teams \\
\hline & Teamevents \\
\hline & Bestenliste \\
\hline & $\begin{array}{l}\text { Netzwerkfunktionen } \\
\text { (Kommentare, Likes) }\end{array}$ \\
\hline & $\begin{array}{l}\text { Glaube an ein über- } \\
\text { geordnetes Ziel (Epic } \\
\text { Meaning) }\end{array}$ \\
\hline
\end{tabular}

Im Bereich der Gesundheitswissenschaften und Medizin wurden einige Spiele entwickelt, die u.a. für das Einüben und Trainieren von praktischen und theoretischen Fertigkeiten genutzt werden (z. B. beim Notfallmanagement; [29, 30]).

Mit Blick auf Serious Games fokussieren die Übersichtsarbeiten von Fleming et al. [31] und Lau et al. [32] vor allem auf psychische Erkrankungen. Bei Lau et al. zeigten 9 von 10 Studien eine Reduktion psychischer Symptome und auch bei Fleming et al. wies die Mehrzahl der identifizierten Studien im Gruppenvergleich auf eine signifikante Symptomreduktion hin. Nguyen und Kollegen [33] fassten die Ergebnisse zu Serious Games bei älteren Menschen ( $>50$ Jahre) zusammen. Bei 18 Studien traten positive Effekte auf, die sich insbesondere in physischen Outcome-Parametern sowie kognitiven Indikatoren zeigten.

Sinnvoll erscheinen Serious Games zur Ansprache von schwer erreichbaren, aber medienaffinen Zielgruppen (wie Personen mit niedrigerem Bildungsstand), die mit den klassischen Lernmedien und -umgebungen schwer $\mathrm{zu}$ erreichen sind. Des Weiteren besteht die
Möglichkeit, durch den gezielten Einsatz von Serious Games in Gesundheitskampagnen Aufmerksamkeit für bestimmte gesundheitsrelevante Themen zu evozieren. Dies gilt vor allem für Zielgruppen, die ansonsten keine Affinität zum Thema haben. Gesundheitsrelevante Inhalte können beispielsweise in bereits bestehende und erfolgreiche Computerspiele unterschwellig im Sinne des Entertainment-Education-Ansatzes integriert werden [11]. Ein weiterer Vorteil des Einsatzes von Spielen ist die kontinuierliche und automatisierte Erhebung von Daten und Feedbackschlaufen, aus denen Möglichkeiten zur Anpassung der Anwendungen, aber auch des HealthMonitorings resultieren. Diese Möglichkeiten erfordern im Gegenzug auch einen kritischen Umgang mit den Datenschutzvorschriften.

Trotz dieser vielfältigen wissenschaftlichen Entwicklungen mangelt es bislang an einer Infrastruktur, die den im Bereich Serious Games und Gamification forschenden und praktisch tätigen Akteuren einen wissenschaftlichen Diskurs und die Vernetzung ermöglichen [24].

\section{Beispiele für Serious Games}

Im Jahr 2006 wurde das Computerspiel „Re-Mission“ veröffentlicht. Ziel des Spiels ist es, das Verhalten und die Einstellung von jungen Patienten bezüglich ihrer Krebserkrankung positiv zu beeinflussen. Die Spielenden fliegen mit ihrer Spielfigur durch den Körper eines virtuellen Patienten, um gegen Tumorzellen und Symptome von Krebserkrankungen zu kämpfen. Eine Studie konnte einen förderlichen Einfluss auf den Umgang mit der eigenen Krankheit (Disease-Management), die Selbstwirksamkeitserwartung, das krankheitsbezogene Wissen sowie das Kommunikationsverhalten der Kinder und Jugendlichen nachweisen [34].

Wie auch in klassischen Aufklärungskampagnen stellt die HIV/Aids-Prävention eine häufig adressierte Thematik von Serious Games dar. Dies reicht von simplen Minispielen wie „Shagland“ (Jump'n'-Run-Spiel, in dem Krankheiten ausgewichen werden muss) und "Catch the Sperm“ (das Einfangen von Spermien mittels eines fliegenden Kondoms) bis hin zu komplexeren Spielinhalten, wie „Interactive Nights Out“, welches an über 200 Soldaten der US-Army verteilt wurde, um das Thema HIV/Aids-Prävention $\mathrm{zu}$ thematisieren [35].

Weitere Serious Games wie „Bronkie the Bronkosaurus“, „Packy and Marlon“ und „Escape from Diab“ wurden zur Verbesserung von krankheitsrelevantem Wissen bei chronischen Krankheiten (Asthma und Diabetes mellitus) eingesetzt und zum Teil positiv evaluiert [36].

Das Serious Game „Boson X“ wurde im Rahmen einer Untersuchung von Personen eingesetzt, die an klinischer Depression erkrankt waren. Ziel des Spiels ist es, durch das Berühren von Feldern Energie zu sammeln, wobei die Figur mit zunehmender Energie schneller wird. Unmittelbar nach sechs Wochen konnte in der Trainingsgruppe eine Verbesserung der kognitiven Fähigkeiten und eine tendenzielle Reduktion des Grübelns nachgewiesen werden [37].

Eine besondere Zielsetzung hat das Serious Game „Outbreak at Watersedge“. In dem Adventure-Spiel muss die Public-Health-Krise in einer Gemeinde gelöst werden. Das Spiel wurde von der School of Public Health der University of Minnesota entwickelt. Es soll die Faszination für das Thema Public Health wecken und den Spielenden überzeugen, die berufliche Zukunft in dem Feld der Gesundheitswissenschaften zu suchen [38].

Im Bereich der medizinischen Ausbildung wurden einige Serious Games entwickelt. Als Beispiel soll „EMERGE“ erwähnt werden, das die Spielenden in die Situation einer virtuellen Notaufnahme versetzt, in der in Echtzeit Patienten behandelt werden müssen. Die Zielsetzung des Spiels ist dabei die Verbesserung der klinischen Entscheidungskompetenz [29, 30, 39].

\section{Gamification zur Prävention und Gesundheitsförderung}

Die Ausrichtung der Forschung im Bereich Gamification entwickelt sich derzeit von Projektberichten hin zu wissenschaftlich fundierten Studien und Reviews. Laut Seaborn und Fels sind die häufigsten Anwendungsgebiete Bildung 
(26\%) und Gesundheit (13\%; [40]). Einem Großteil der Studien fehlt jedoch der Bezug zu theoretischen Grundlagen und sie sind häufig nicht präzise bei der Differenzierung der unterschiedlichen spielerischen Ansätze [10, 41]. Das Anwendungs- und Forschungsfeld ist sehr divers und interprofessionell aufgestellt, was zu einer fragmentierten Wissensbasis geführt hat [42].

Die Verwendung von Gamification für gesundheitliche Zwecke ist verbreitet und wird als vielversprechender Ansatz zur Förderung des Gesundheitsverhaltens und seiner Determinanten eingeschätzt $[42,43]$. Studien konnten zeigen, dass sich Gamification positiv auf Motivation und Leistung auswirken kann und $\mathrm{zu}$ einem langfristigen Engagement (z. B. im Bereich Lernen) der Nutzenden führen kann $[23,44]$. Darüber hinaus wird erwartet, dass die Erfüllung der psychologischen Bedürfnisse das Wohlbefinden steigert [12].

Gamification kann in zwei verschiedenen Gesundheitsbereichen eingesetzt werden: im Bereich der Unterstützung von Intervention mit dem Ziel von gesundheitsrelevanten Verhaltensänderungen und im Bereich des Lernens, wie beispielsweise bei der Ausbildung in Gesundheitsberufen oder bei der Bereitstellung von Lerneinheiten für gesundheitliche Themen. Diese beiden Schwerpunkte sollen im Folgenden näher skizziert werden.

\section{Gesundheitsrelevante Verhaltensänderungen}

Viele spielbasierte Anwendungen zielen auf die Förderung der körperlichen Gesundheit, die Rehabilitation chronischer Krankheiten [23, 42] sowie auf die Förderung der psychosozialen Gesundheit ab. In einem systematischen Review von Sardi et al. legte die Hälfte der Veröffentlichungen den Fokus auf den Umgang von Patienten mit chronischen Erkrankungen bzw. die Rehabilitation $(n=23)$, gefolgt von der Förderung körperlicher Aktivität $(n=15)$. Insgesamt 8 Anwendungen bezogen sich auf den Bereich psychische Gesundheit. Insgesamt waren Feedback, Belohnungen, Fortschrittsanzeigen und soziale Interaktion die am häufigsten verwendeten Spielmechaniken [45].

In der Übersichtsstudie von Johnson et al. belegen $59 \%$ der einbezogenen Studien positive und $41 \%$ heterogene Effekte. Häufig wurden die Wirkungen von Gamification auf das Gesundheitsverhalten und eher selten die Wirkungen auf Emotionen und Stimmungen untersucht. Dass Gamification oftmals im Feld der psychischen Gesundheit eingesetzt wird, liegt wahrscheinlich nicht zuletzt daran, dass die theoretischen Grundlagen von Wohlbefinden und Gamification nah beieinander zu liegen scheinen [46].

Insbesondere im Bereich der körperlichen Gesundheit lässt sich eine Vielzahl von Interventionen verzeichnen, bei denen auf Elemente der Gamification zurückgegriffen wird. Koivisto und Hamari untersuchten in einem systematischen Literaturreview den Einsatz von Gamification im Bereich physischer Aktivität und konnten durchaus positive Effekte identifizieren. Sie mahnten aber auch die teilweise qualitativ schlechte Ausführung der Studien an [47]. Ein Großteil der Anwendungen mit Gamification ist im kommerziellen Bereich zu finden, wie zum Beispiel in Lauf-Apps wie "Zombie Run“ (hier wird die joggende Person von virtuellen Zombies verfolgt und absolviert so ein Intervalltraining).

\section{Lernen mit Gamification}

Der Einsatz von Gamification in Bildungskontexten gehört zu den am stärksten repräsentierten Forschungsbereichen innerhalb der Gamificationliteratur. Häufige Ziele von Gamification im Bildungskontext sind die Förderung von unterschiedlichen Wissensarten, die Steigerung von (Lern-)Motivation und der Erwerb von Fähigkeiten und Kompetenzen [17]. Trotz der oftmals fehlenden theoretischen Fundierung empirischer Studien zum Thema Gamification und Lernerfolg, die häufig im Zentrum von Kritik steht [13], gibt es theoretische Ansätze, die sich den Effekten von Gamification auf den Lernerfolg widmen, wie zum Beispiel die Theory of Gamified Learning [48]. Diese Theorie geht davon aus, dass Spieldesignelemente nicht direkt auf den Lernerfolg wirken, sondern lediglich lernrelevante Faktoren fördern können, die wiederum einen positiven Zusammenhang mit dem Lernerfolg aufweisen. $\mathrm{Zu}$ solchen lernrelevanten Faktoren kann eine tiefere kognitive Verarbeitung gezählt werden oder auch die Dauer, die Lernende mit dem Lernmaterial verbringen [48]. Basierend auf dieser Theorie kann Gamification zum Lernerfolg beitragen.

Eine Metaanalyse von Sailer und Homner [17] untersuchte die Wirkung von Gamification auf lernrelevante Ergebnisse und fand hierbei positive, kleine Effekte (k) von Gamification auf kognitive $(k=19)$, motivationale $(k=16)$ und verhaltensbezogene Lernergebnisse $(k=9)$. Moderatoranalysen zeigen, dass vor allem die Verwendung von narrativen Komponenten, wie beispielsweise Geschichten oder über den Verlauf entwickelbare Avatare, besonders effektiv für die Förderung verhaltensbezogener Lernleistungen sein können. Während Wettbewerb zwischen einzelnen Lernern nicht zwangsläufig zu positiven Effekten führt, zeigen Kombinationen von Wettbewerb und Zusammenarbeit, beispielsweise Teambestenlisten, bei denen Gruppen von Lernern gegeneinander antreten, positive Effekte auf verhaltensbezogene Outcomes sowie auch - zumindest in manchen Analysen - auf die Motivation. Auch allgemein im Bildungsbereich und speziell in der Metaanalyse zeigt sich ein hoher Anteil an Untersuchungen mit geringer bis moderater Studienqualität $[17,49]$.

Der Einsatz von Gamification in speziellen Domänensettings, wie zum Beispiel im Schulunterricht oder in der medizinischen Ausbildung, zeigt ebenfalls positive Ergebnisse [50, 51].

\section{Gamification zur Förderung des Wohlbefindens}

Als deutschsprachiges Beispiel für den Einsatz von Gamification in der psychischen Gesundheitsförderung soll „ONYA - The Wellbeing Game“ dargestellt werden. Diese Anwendung basiert auf dem von der Mental Health Foundation Neuseeland entwickelten „The Wellbeing Game" (TWBG; [52]) und wurde an der Leuphana Universität Lüneburg 
\begin{tabular}{ll}
\hline Vernetzen & $\begin{array}{l}\text { Tragfähige soziale Beziehungen sind von Bedeutung für das Wohlbefinden. Dazu gehören neben den engeren sozialen Netzwerken } \\
\text { (Familie, Freunde) auch Bekanntschaften aus dem Arbeitsumfeld oder dem kommunalen Raum. Das Wohlbefinden wird umso mehr } \\
\text { gefördert, je stärker das soziale Umfeld und somit die soziale Unterstützung ist }\end{array}$ \\
Bewegen & $\begin{array}{l}\text { Körperliche Aktivität ist seit Langem als positiver Einflussfaktor auf die psychische Gesundheit und das Wohlbefinden bekannt. } \\
\text { Unter körperlicher Aktivität wird sowohl Sport als auch alltägliche Bewegung während der Freizeit und im beruflichen Kontext ver- } \\
\text { standen }\end{array}$ \\
Achtsam sein & $\begin{array}{l}\text { Achtsamkeit spielt eine relevante Rolle für das Wohlbefinden. Darunter versteht man die bewusste Wahrnehmung und das wertfreie } \\
\text { Erleben des aktuellen Moments mit Gedanken, Gefühlen und Sinneseindrücken. Dabei handelt es sich um einen klaren Bewusst- } \\
\text { seinszustand, in dem alle inneren und äußeren Eindrücke vorurteilsfrei registriert werden }\end{array}$ \\
Lernen & $\begin{array}{l}\text { Lernen und die Bewältigung von Herausforderungen sind für das Wohlbefinden von Bedeutung. Lernsituationen und Herausfor- } \\
\text { derungen lassen sich u. a. im Arbeitskontext (z. B. Verantwortungsbereiche erweitern, Fortbildung besuchen) und im Privatleben } \\
\text { finden (z. B. eine neue Sprache erlernen, neue Sportart ausprobieren) }\end{array}$ \\
Geben in Form von Hilfsbereitschaft und prosozialem Verhalten sind relevant für das Wohlbefinden. Die Lebenszufriedenheit kann \\
Gurch altruistisches und uneigennütziges Verhalten (Spenden, Ausübung eines Ehrenamts, kleine Gefälligkeiten im Alltag) gestei- \\
gert werden
\end{tabular}

mit Unterstützung der Bundeszentrale für gesundheitliche Aufklärung (BZgA) für den deutschsprachigen Kontext adaptiert und evaluiert [53]. Die Anwendung basiert auf dem Konzept der Five Ways to Wellbeing. Den Kern dieses evidenzbasierten Ansatzes bilden 5 Dimensionen zur Förderung des Wohlbefindens ([54];

\section{- Tab. 2).}

Die Five Ways to Wellbeing zielen auf eine Sensibilisierung und individuelle Verhaltensänderung ab. Die Kommunikationsbotschaften ermöglichen einen niedrigschwelligen und im Alltag leicht integrierbaren Zugang.

Die zentrale Idee im webbasierten Spiel "ONYA“ ist die Dokumentation von Aktivitäten zur Förderung des Wohlbefindens und deren Zuordnung zu den Five Ways to Wellbeing (• Abb. 2). Während der Anwendung von „ONYA“ sollen Spielende für Aktivitäten sensibilisiert werden, die förderlich für das Wohlbefinden sein können. Über den niedrigschwelligen Zugangsweg der Dokumentation von Alltagsaktivitäten sollen die Nutzenden langfristig motiviert werden, diese vermehrt auszuführen und bewusster wahrzunehmen. Vergleichbar mit einem Onlinetagebuch können die Nutzenden alleine oder im Team alltägliche Aktivitäten in der Webapplikation erfassen. Dafür werden die Aktivitäten einem oder mehreren Faktoren des Wohlbefindens zugeordnet.

Zur Förderung der Nutzungsmotivation werden im Rahmen von ONYA diverse Spieldesignelemente eingesetzt. Für jede erfasste Aktivität erhalten die Spielenden Punkte. Eine Übersicht über den aktuellen Punktestand bietet eine Statusanzeige, die außerdem das integrierte Levelsystem darstellt. Die Nutzenden erhalten über diese Funktion ein direktes Feedback zu ihrem Spielstand und die Möglichkeit, eine bessere Selbsteinschätzung vorzunehmen.

Das Kompetenzerleben wird als wesentliche Dimension der psychologischen Grundbedürfnisse nach Ryan und Deci [20] über das Vergeben von Auszeichnungen (Badges) angesprochen (• Abb. 3). Diese werden für das Erreichen von definierten Spielzielen vergeben, wie z.B. 10 dokumentierte Stunden in der Kategorie „Bewegung“ oder ein geplantes Teamevent. Als zusätzliche Belohnung schalten die Spielenden bei Erreichen eines neuen Levels Grafiken frei, die gleichzeitig Unterhaltung und informative Fakten zu gesundheitsrelevanten Themen bieten (Infotainments). Die Nutzer erhalten Vorschläge für Aktivitäten in Form von Aufgaben (Quests), die mit voranschreitendem Spielfortschritt in Anlehnung an die Bloom'sche Taxonomie an Komplexität zunehmen [55]. Spielende können eigene Teams anlegen oder bestehenden Teams beitreten. Innerhalb dieser Teams können gemeinsame Aktivitäten geplant und dokumentiert werden. Alle Spielenden bzw. Teams werden in einer Bestenliste aufgeführt. Über die Funktion „Inspiration“ werden Vorschläge für weitere Aktivitäten für Wohlbefinden gegeben.
Das Autonomieerleben der Spielenden wird über die Möglichkeit der Gestaltung eines eigenen Profils sowie über die freie Wahl und Gestaltung der Aktivitäten angesprochen. Die erste Studie wird derzeit ausgewertet, erste Hinweise lassen auf einen Wissenserwerb, eine Verbesserung des Wohlbefindensstatus und eine Assoziation mit den psychologischen Bedürfnissen nach Ryan und Deci sowie eine hohe Affinität zu den Spieldesignelementen schließen.

\section{Vor- und Nachteile von spielerischen Ansätzen in der Prävention und Gesundheitsförderung}

Bei der Betrachtung der oben beschriebenen spielerischen Anwendungen lassen sich verschiedene Vor- und Nachteile von Serious Games und Gamification in der Prävention und Gesundheitsförderung ableiten.

Durch den Einsatz von Serious Games können Themen an die Spielenden herangetragen werden, die ansonsten in deren Lebenswelten auf wenig Aufmerksamkeit stoßen würden. Gesundheitsrelevante Themen können dabei sowohl in eigens entwickelten Serious Games vermittelt oder in bereits bestehende Spiele unterschwellig implementiert werden. Der Einsatz von Serious Games ist zudem dann sinnvoll, wenn komplexere Inhalte über einen längeren Zeitraum avisiert werden, wie zum Beispiel Prozesse zur Verhaltensänderung. Serious Games 


\section{Leitthema}

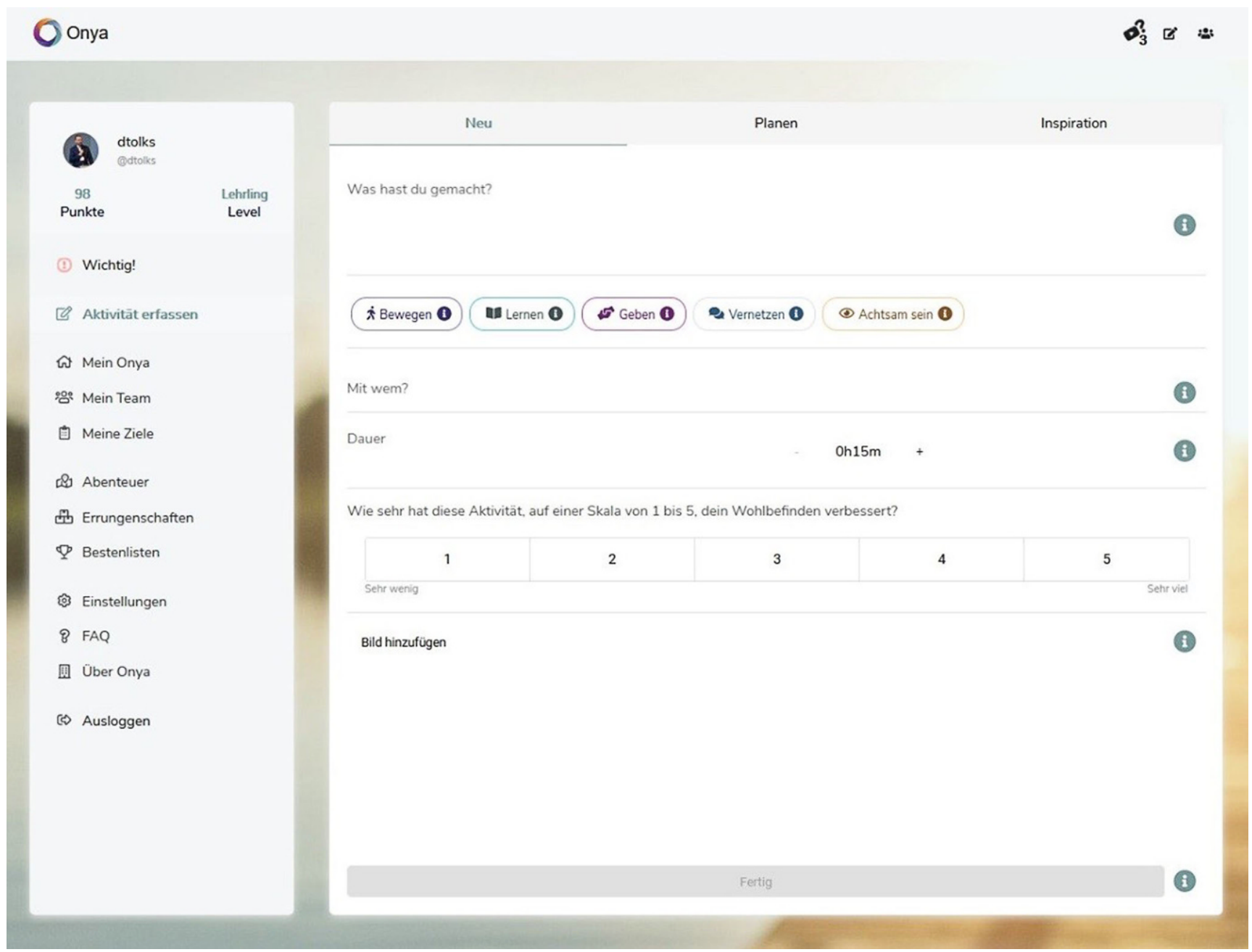

Abb. 2 ム Erfassung von Aktivitäten zum Wohlbefinden in der spielbasierten Anwendung "ONYA“

können die Aufmerksamkeit erzeugen und erhalten, was eine zentrale Voraussetzung für eine effektive und nachhaltige Verhaltensänderung ist [36]. Komplexere Zusammenhänge und evtl. Verhaltensund Einstellungsänderungen sind eher möglich. Serious Games haben zudem die Vorteile des Mediums Spiel, wie den Immersionsfaktor sowie die Möglichkeit des Flowerlebens [8].

Der große Vorteil von Gamification ist die universelle Einsatzmöglichkeit. Gamification kann theoretisch in jedem nichtspielerischen Kontext eingesetzt werden. Für die Zwecke der Gesundheitsförderung und Prävention ist Gamification insbesondere dort sinnvoll, wo Lernen unterstützt, Motivation erhöht und Verhaltensänderung evoziert werden kann. Der Einsatz von Gamification ist speziell dort geeignet, wo die grundsätzliche Motivation der Nut- zenden eher niedrig ist. Aktivitäten, die von den Nutzenden als eher langweilig, eintönig und sogar störend empfunden werden, können durch den Einsatz von Spieldesignelementen einer höheren Bedeutsamkeit zugesprochen werden. Weitere Vorteile werden in - Tab. 3 dargestellt.

Die Nachteile bei dem Einsatz von Gamification liegen v. a. in den unterschiedlichen Zielgruppen begründet. Es gibt verschiedene Spielertypen, Unterschiede zwischen den Geschlechtern (Gendergap) sowie eine niedrigere Nutzung von Gamification im Alter. Zudem besteht die Gefahr, dass der Einsatz von Gamification nicht als unterstützendes Element fungiert, sondern als zentraler Aspekt der Anwendung wahrgenommen wird und die eigentlichen Inhalte in den Hintergrund treten. Durch den Einsatz von Gamification wird zudem die Ten- denz zur individualisierten Gesundheitsförderung verstärkt, die Strukturen und Institutionen treten dabei in den Hintergrund.

\section{Ausblick}

Die positive Entwicklung von Serious Games for Health und der Einsatz von Spieldesignelementen in der Prävention und Gesundheitsförderung lassen auf eine weitere Entwicklung und viele Einsatzszenarien hoffen. Für die zukünftige Entwicklung ist es insbesondere wichtig, dass die Akteure dieses Anwendungs- und Forschungsfeldes interdisziplinär und interprofessionell zusammenarbeiten. Bislang mangelt es an einer Infrastruktur, die den im Bereich Serious Games und Gamification forschenden und praktisch tätigen Akteuren einen wissenschaftlichen Diskurs und die Vernetzung er- 


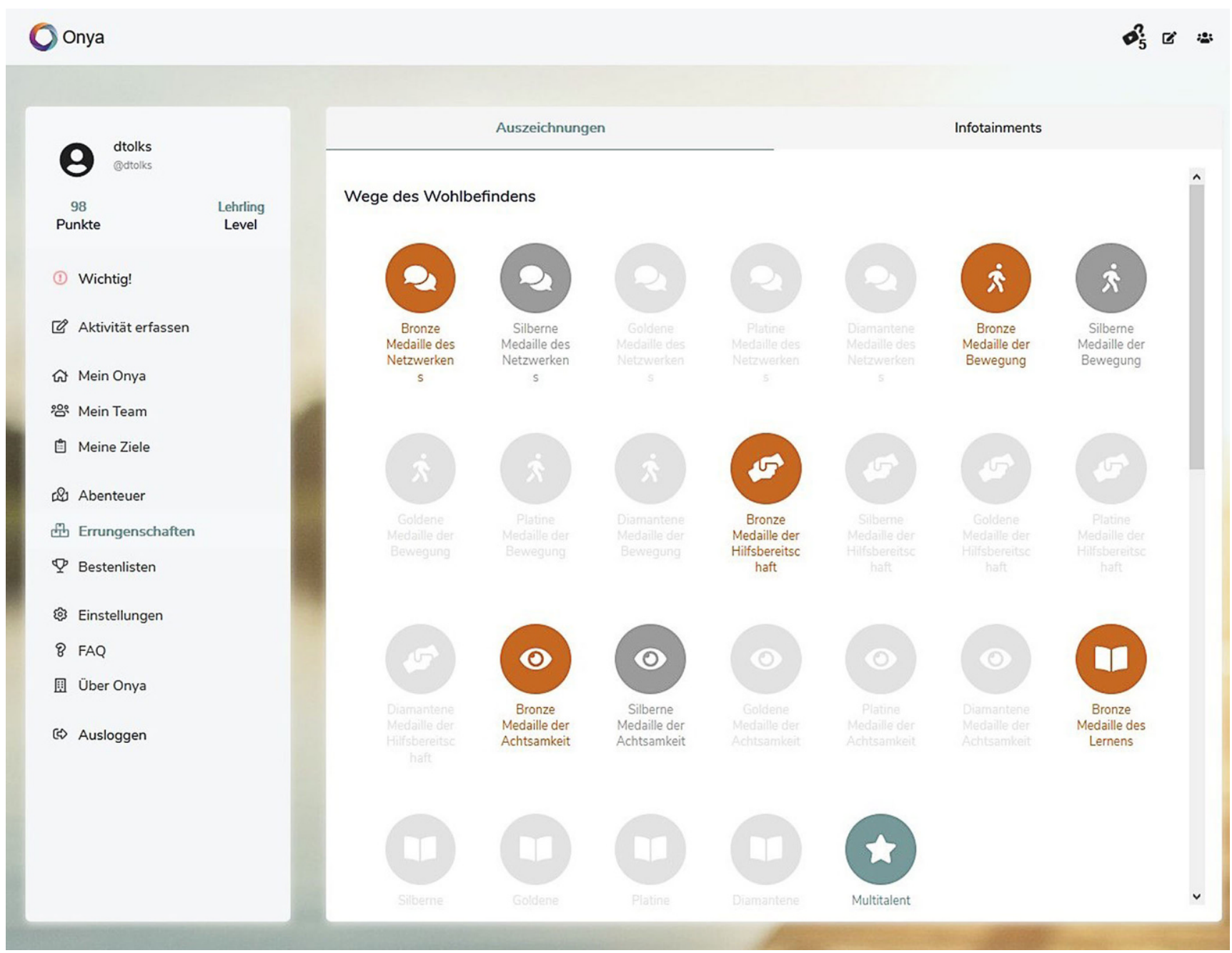

Abb. 3 ॥ Darstellung von Auszeichnungen (Badges) in der spielbasierten Anwendung „ONYA"

möglichen [24]. Um diese Entwicklung zu forcieren, wurde im Oktober 2019 das „Netzwerk Serious Games und Gamification for Health“" gegründet. Dieses Netzwerk wird als Community Working Group des Hochschulforums Digitalisierung gefördert und hat als Ziel, eine Website und Datenbank mit den aktuellen Forschungsergebnissen, Anwendungsszenarien und Projekten zu erstellen und vor allem die Akteure in diesem Feld zusammenzubringen [56].

Es bedarf zudem, wie bei allen neuen Forschungsfeldern, einer weiteren Grundlagenforschung. Dabei bleibt es von allerhöchster Wichtigkeit, die Taxonomie der verschiedenen spielerischen Anwendungen in der Forschung zu beachten und evidenzbasierte Erkenntnisse $\mathrm{zu}$ generieren. Auch die Differenzierung der einzelnen Elemente und deren
Zusammenspiel sind noch nicht ausreichend beforscht. Im Bereich Serious Games zeigt sich ebenfalls Forschungsbedarf bezüglich der Implementierung von Lern- und Gametheorien sowie didaktischen Methoden. Auch dort zeigen sich Unklarheiten bezüglich der Wirkungsweisen von Spiel- und Lerneinheiten.

Im Feld der digitalen Spiele ist zudem die rasant fortschreitende technische Weiterentwicklung zu berücksichtigen. Die technologische Leistungsfähigkeit in der Computer- und Konsolenentwicklung wurde seit jeher von den Ansprüchen an die Spiele gefördert. Interessant wird zukünftig sein, welche neuen Technologien sich durchsetzen werden und welche Möglichkeiten sich daraus für spielerische Anwendungen der Gesundheitsförderung und Präven- tion ergeben. Dabei sind die Wechsel der Eingabegeräte (vom Controller hin zur Sprachsteuerung) und Darstellungsformen (vom Bildschirm hin zur erweiterten und virtuellen Realität) auch für die Entwicklung von Serious Games und gamifizierten Anwendungen relevant.

Betrachtet man die derzeitigen Entwicklungen, insbesondere im Bereich Gamification, entsteht schnell der Eindruck eines Hypes. Daher stellt sich die Frage, inwieweit es Serious Games und Gamification auch zukünftig noch geben wird. Laut dem Gartner Hype Cycle for Education hat Gamification bereits die ersten drei Stufen des Hypeprozesses durchlaufen und befindet sich in der Konsolidierungs- und Implementierungsphase [57]. Da die Spieldesignelemente auf die fundamentalen Prozesse der Motivation abzielen, ist es 
Tab. 3 Vor- und Nachteile von Gamification

\begin{tabular}{l|l}
\hline Vorteile & Nachteile \\
\hline $\begin{array}{l}\text { Fördert die Motivation [12, 21, 44] } \\
\text { Fördert das Userengagement [13, 19] }\end{array}$ & $\begin{array}{l}\text { Unterschiedliche Nutzertypen [13] } \\
\text { Nutzung von Gamification wird mit zunehmendem } \\
\text { Alter schwieriger [61] } \\
\text { Unterschiede zwischen den Geschlechtern (Gender- } \\
\text { Gap; [61]) }\end{array}$ \\
\hline Fördert positiven Wettbewerb [59] & $\begin{array}{l}\text { Gefahr eines übertriebenen Einsatzes von Gamifica- } \\
\text { tion („,Übergamification“) }\end{array}$ \\
\hline $\begin{array}{l}\text { Ermöglicht Lernen aus Fehlern (Freedom } \\
\text { to Fail) }\end{array}$ & $\begin{array}{l}\text { Möglichkeit des Novelty Effects (nur anfängliche } \\
\text { Leistungssteigerung bei Einführung einer neuen }\end{array}$ \\
\hline $\begin{array}{l}\text { Ist leicht zugänglich [43] } \\
\text { Ist bereits durch das Medium Spiel be- } \\
\text { kannt [2] }\end{array}$ & $\begin{array}{l}\text { Tendenz der Individualisierung und fehlende } \\
\text { Berücksichtigung der Verhältnisse und Strukturen }\end{array}$ \\
\hline $\begin{array}{l}\text { Lässt sich in den Alltag integrieren } \\
\text { Ist kosteneffizient [60] } \\
\text { Kann das Wohlbefinden fördern [23, 52] }\end{array}$ & \\
\hline
\end{tabular}

ebenfalls wahrscheinlich, dass auch in Zukunft der Einsatz von Gamification gute Effekte erzielen wird.

Der Einsatz von Serious Games und Gamification im Feld von Prävention und Gesundheitsförderung wird sich sicherlich weiterentwickeln. Das Medium Spiel ist seit Beginn der Menschheitsgeschichte integraler Bestandteil unserer Kultur und wird auch weiterhin einen großen Anteil unseres Alltags einnehmen. Die positiven Ergebnisse bei dem Einsatz von Serious Games und Gamification im Bereich der Verhaltens- und Einstellungsänderung sowie im Bereich Lernen und im therapeutischen Einsatz lassen auch die zukünftige Entwicklung als sehr Erfolg versprechend erscheinen.

Auch die neuen Entwicklungen im Gesundheitswesen, insbesondere das digitale Versorgungsgesetz, eröffnen neue Forschung- und Anwendungsfelder. Das Feld der Gesundheitsförderung und Prävention könnte hier sogar als fortschrittlicher Innovator im Gesundheitswesen fungieren.

Durch die zunehmend reflektierte und wissenschaftlich fundierte Diskussion über Computerspiele ist die Wahrscheinlichkeit gestiegen, dass in Zukunft neue Projekte leichter zu realisieren sein werden, was einen weiteren Anschub und neue Forschungsvorhaben zur Folge haben könnte. Bill Gates hatte unlängst im Rahmen einer Paneldiskussion in New York seine Vision des Lernens in der Zukunft beschrieben, in der Serious Games einen integralen Bestandteil des Wissens- und Kompetenzerwerbs darstellen: „Instead of just reading a chapter on solving equations ..., watch a superengaging video that shows you how it's done, and play a game that reinforces the concepts ..."

\section{Korrespondenzadresse}

\section{Dr. Daniel Tolks}

Zentrum für Angewandte Gesundheitswissenschaften, Leuphana Universität

Lüneburg

Wilschenbrucher Weg 84 a, 20335 Lüneburg,

Deutschland

daniel.tolks@leuphana.de

Funding. Open Access funding provided by Projekt DEAL.

\section{Einhaltung ethischer Richtlinien}

Interessenkonflikt. D. Tolks, C. Lampert, K. Dadaczynski, E. Maslon, P. Paulus und M. Sailer geben an, dass kein Interessenkonflikt besteht.

Für diesen Beitrag wurden von den Autoren keine Studien an Menschen oder Tieren durchgeführt. Für die aufgeführten Studien gelten die jeweils dort angegebenen ethischen Richtlinien.

Open Access. Dieser Artikel wird unter der Creative Commons Namensnennung 4.0 International Lizenz veröffentlicht, welche die Nutzung, Vervielfältigung, Bearbeitung, Verbreitung und Wiedergabe in jeglichem Medium und Format erlaubt, sofern Sie den/die ursprünglichen Autor(en) und die Quelle ordnungsgemäß nennen, einen Link zur Creative Commons Lizenz beifügen und angeben, ob Änderungen vorgenommen wurden.

Die in diesem Artikel enthaltenen Bilder und sonstiges Drittmaterial unterliegen ebenfalls der genannten Creative Commons Lizenz, sofern sich aus der Abbildungslegende nichts anderes ergibt. Sofern das betreffende Material nicht unter der genannten Creative Commons Lizenz steht und die betreffende Handlung nicht nach gesetzlichen Vorschriften erlaubt ist, ist für die oben aufgeführten Weiterverwendungen des Materials die Einwilligung des jeweiligen Rechteinhabers einzuholen.

Weitere Details zur Lizenz entnehmen Sie bitte der Lizenzinformation auf http://creativecommons.org/ licenses/by/4.0/deed.de.

\section{Literatur}

1. Dadaczynski K, Schiemann S, Paulus P (2016) Gesundheit spielend fördern: Potenziale und Herausforderungen von digitalen Spieleanwendungen für die Gesundheitsförderung und Prävention. Beltz Juventa, Weinheim, Basel

2. Tolks D, Dadaczynski K, Horstmann D (2018) Einführung in die Vergangenheit, Gegenwart und Zukunft von Serious Games (for Health) Präv Gesundheitsf 13:272-279. https://doi.org/10 1007/s11553-018-0667-9

3. Abt CC (1970) Serious games. Viking Press, New York

4. Sawyer B, Smith P (2008) Serious Games Taxonomy. Serious Games Summit at the Game Developers Conference, San Francisco, CA

5. Sostmann K, Tolks D, Fischer M, Buron S (2010) Serious Games for Health: Spielend lernen und heilen mit Computerspielen? GMS Med Inform Biom Epidemiol 6:1-8. https://doi.org/10.3205/ mibe000112

6. Gauthier A, Kato PM, Bul KCM et al (2019) Board games for health: a systematic literature review and meta-analysis. Games Health J 8:85-100. https://doi.org/10.1089/g4h.2018.0017

7. Tolks D, Lampert C (2016) Abgrenzung von Serious Games zu anderen Lehr- und Lernkonzepten. In: Dadaczynski K, Schiemann S, Paulus P (Hrsg) Gesundheit spielend fördern. Potenziale und Herausforderungen von digitalen Spieleanwendungen für die Gesundheitsförderung und Prävention. Beltz Juventa, Weinheim, S 191-217

8. Wang H, Singhal A (2014) Digital games: the SECRET of alternative health realities. In: Kim DK, Singhal A, Kreps GL (Hrsg) Health communication. Strategies for developing global health programs. Peter Lang, New York, S67-79

9. Clark DB, Tanner-Smith EE, Killingsworth SS (2016) Digital games, design, and learning: a systematic review and meta-analysis. RevEduc Res 86:79-122. https://doi.org/10.3102/0034654315582065

10. Baranowski T (2018) Games for health research-past, present, and future. Präv Gesundheitsf 13:333-336. https://doi.org/10.1007/ s11553-018-0657-y

11. Lampert C (2007) Gesundheitsförderung im Unterhaltungsformat. Nomos, Baden Baden

12. Ryan RM, Rigby CS, Przybylski A (2006) The motivational pull of video games: a self-determination theory approach. Motiv Emot 30:344-360

13. Hamari J, Koivisto J, Sarsa H (2014) Does gamification work? A literature review of empirical studies on gamification. In: Proceedings of the 
47th Hawaii International Conference on System Sciences Hawaii

14. Deterding S, Dixon D, Khaled R, Nacke L (2011) From game design elements to gamefulness: defining gamification. In: Proceedings of the 15th international academic MindTrek conference: Envisioning future media environments. ACM, Tampere, S9-15

15. Werbach K, Hunter D (2012) For the win: how game thinking can revolutionize your business. Wharton Digital Press, Philadelphia

16. Landers RN, Auer EM, Collmus AB, Armstrong MB (2018) Gamification Science, its history and future: definitions and a research agenda. Simul Gaming 49:315-337. https://doi.org/10.1177/ 1046878118774385

17. Sailer M, Homner L (2019) The gamification of learning: a meta-analysis. Educ Psychol Rev. https://doi.org/10.1007/s10648-019-09498-w

18. Deterding S (2012) Gamification: designing for motivation. Interactions 19:14-17

19. Sailer M (2016) Die Wirkung von Gamification auf Motivation und Leistung: Empirische Studien im Kontext manueller Arbeitsprozesse. Springer, Wiesbaden

20. Ryan RM, Deci EL (2000) Self-determination theory and the facilitation of intrinsic motivation, social development, and well-being. Am Psychol 55:68

21. Xi N, Hamari J (2019) Does gamification satisfy needs? A study on the relationship between gamification features and intrinsic need satisfaction. Int JInfManag 46:210-221. https://doi.org/10.1016/j. ijinfomgt.2018.12.002

22. Lampert C, Schwinge C, Tolks D (2009) Der gespielte Ernst des Lebens: Bestandsaufnahme und Potenziale von Serious Games (for Health). MedienPädagogik 15/16:16. https://doi.org/10. 21240/mpaed/15+16/2009.03.11.X

23. Johnson D, Deterding $S$, Kuhn K-A et al (2016) Gamificationforhealth and wellbeing: a systematic review of the literature. Internet Interv 6:89-106

24. Dadaczynski K, Tolks D (2018) Spielerische Ansätze als innovative Kommunikationsstrategie der Gesundheitsförderung und Prävention. Präv Gesundheitsf 13:269-271. https://doi.org/10. 1007/s11553-018-0672-z

25. Lampert C, Tolks D (2016) Grundtypologie von digitalen Spieleanwendungen im Bereich Gesundheit. In: Dadaczynski K, Schiemann S, Paulus P (Hrsg) Gesundheit spielend fördern. Potenziale und Herausforderungen von digitalen Spieleanwendungen für die Gesundheitsförderung und Prävention. Beltz Juventa, Weinheim, S218-233

26. Lu AS, Kharrazi H (2018) A state-of-the-art systematic content analysis of games for health. Games Health J 7:1-15. https://doi.org/10.1089/ g4h.2017.0095

27. DeSmet A, Van Ryckeghem D, Compernolle $S$ et al (2014) A meta-analysis of serious digital games for healthy lifestyle promotion. Prev Med 69:95-107. https://doi.org/10.1016/j.ypmed.2014.08.026

28. Connolly TM, Boyle EA, MacArthur E et al (2012) A systematic literature review of empirical evidence on computer games and serious games. Comput Educ 59:661-686. https://doi.org/10. 1016/j.compedu.2012.03.004

29. Chon S-H, Timmermann F, Dratsch T et al (2019) Serious games in surgical medical education: a virtual emergency department as a tool for teaching clinical reasoning to medical students. JMIR Serious Games 7:e13028. https://doi.org/10. 2196/13028

30. Tolks D, Fischer MR (2013) Serious Games for Health - ernstzunehmende didaktische Konzepte in der medizinischen Ausbildung? GMS Med Inform Biom Epidemiol.https://doi.org/10.3205/mibe000131

31. Fleming TM, Bavin L, Stasiak K et al (2017) Serious games and gamification for mental health: current status and promising directions. Front Psychiatry 7:215. https://doi.org/10.3389/fpsyt.2016.00215

32. Lau HM, Smit JH, Fleming TM, Riper H (2017) Serious games for mental health: are they accessible, feasible, and effective? A systematic review and meta-analysis. Front Psychiatry 7:209. https://doi.org/10.3389/fpsyt.2016.00209

33. Nguyen TTH, Ishmatova D, Tapanainen $T$ et al (2017) Impact of serious games on health and well-being of elderly: a systematic review. In: Proceedings of the 50th Hawaii International Conference on System Sciences Waikoloa Village, Hawaii, USA

34. Kato PM, Cole SW, Bradlyn AS, Pollock BH (2008) A video game improves behavioral outcomes in adolescents and young adults with cancer: a randomized trial. Pediatrics 122:e305-317.https:// doi.org/10.1542/peds.2007-3134

35. Michael D, Chen S (2006) Serious games: games that educate, train, and inform. Thomson Course Technology, Boston

36. Baranowski T, Buday R, Thompson D, Baranowski J (2008) Playing for real: video games and stories for health-related behavior change. Am J Prev Med 34:74-82. https://doi.org/10.1016/j.amepre.2007. 09.027

37. Kühn S, Kugler DT, Schmalen K et al (2019) Does playing violent video games cause aggression? A longitudinal intervention study. Mol Psychiatry 24:1220-1234

38. University of Minnesota (2004) Outbreak at Watersedge. http://www.mclph.umn.edu/watersedge/ credits.html.Zugegriffen: 30 . März 2020

39. Middeke A, Anders S, Schuelper M et al (2018) Training of clinical reasoning with a serious game versus small-group problem-based learning: a prospective study. PLoS ONE 13:e203851. https:// doi.org/10.1371/journal.pone.0203851

40. Seaborn K, Fels DI (2015) Gamification in theory and action: a survey. Int J Hum Comput Stud 74:14-31

41. Breuer J, Tolks D (2018) Grenzen von "Serious Games for Health“. Präv Gesundheitsf 13:327-332. https://doi.org/10.1007/s11553-018-0654-1

42. Schmidt-Kraepelin M, Thiebes A, Baumsteiger S, Sunyaev D (2018) State of play: a citation network analysis of healthcare gamification studies. In: Research papers, Portsmouth, UK, S 1-17. http:// ecis2018.eu/published-ecis-2018-papers/

43. King D, Greaves F, Exeter C, Darzi A (2013) "Gamification": influencing health behaviours with games. J R Soc Med 106:76-78. https://doi. org $/ 10.1177 / 0141076813480996$

44. Sailer M, Hense JU, Mayr SK, Mandl H (2017) How gamification motivates: an experimental study of the effects of specific game design elements on psychological need satisfaction. Comput Hum Behav 69:371-380. https://doi.org/10.1016/j.chb. 2016.12.033

45. Sardi L, Idri A, Fernández-Alemán JL (2017) A systematic review of gamification in e-Health. J Biomed Inform 71:31-48. https://doi.org/10. 1016/j.jbi.2017.05.011

46. Jones BA, Madden GJ, Wengreen HJ (2014) The FIT Game: preliminary evaluation of a gamification approach to increasing fruit and vegetable consumption in school. Prev Med 68:76-79

47. Koivisto J, Hamari J (2019) Gamification of physical activity: a systematic literature review of comparison studies. In: GamiFIN Levi, Finland, S106-117

48. Landers RN, Landers AK (2014) An empirical test of the theory of gamified learning: the effect of leaderboards on time-on-task and academic performance. Simul Gaming 45:769-785. https:// doi.org/10.1177/1046878114563662

49. Dichev C, Dicheva D (2017) Gamifying education: what is known, what is believed and what remains uncertain: a critical review. Int J Educ Technol High Educ 14:9. https://doi.org/10.1186/s41239-0170042-5

50. Sailer M, Tolks D, Mandl H (2019) Potenziale von Gamification: Empirische Befunde zum Einsatz in Schule und Unterricht. Comput Unterr 115:8-11

51. Nevin CR, Westfall AO, Rodriguez JM et al (2014) Gamification as a tool for enhancing graduate medical education. Postgrad Med J. https://doi. org/10.1136/postgradmedj-2013-132486

52. Green J (2013) Evaluation of the 2012 wellbeing game. Canterbury District Health Board, Canterbury

53. Tolks D, Sailer M, Dadaczynski K et al (2019) ONYA - the wellbeing game: how to use gamification to promote wellbeing. Information 10:58. https:// doi.org/10.3390/info10020058

54. Aked J, Marks N, Cordon C, Thompson S (2008) Five ways to well-being. A report presented to the Foresight Project on communicating the evidence base for improving people's well-being. New Economics Foundation, London

55. Anderson LW, Krathwohl DR, Airasian PW et al (2013) A taxonomy for learning, teaching, and assessing. Pearson New International Edition: a revision of Bloom's taxonomy of educational objectives, abridged edition. Pearson Education, Harlow

56. Hochschulforum Digitalisierung (2020) Community Working Group "Netzwerk Serious Games und Gamification for Health.". https:// hochschulforumdigitalisierung.de/de/cwgseriousgameshealth.Zugegriffen:30. März 2020

57. Gartner Research (2016) Hype cycle for education. https://www.gartner.com/en/documents/ 3364119/hype-cycle-for-education-2016. Zugegriffen: 1. März 2020

58. Horstmann D, Tolks D, Dadaczynski K, Paulus $P$ (2018) Förderung des Wohlbefindens durch "Gamification ". Präv Gesundheitsf 13:305-311. https://doi.org/10.1007/s11553-018-0659-9

59. Kapp KM (2012) The gamification of learning and instruction: game-based methods and strategies for training and education. John Wiley \& Sons, San Francisco, CA

60. Munson S, Poole E, Perry D, Peyton T (2015) Gamification and health. In: Walz S (Hrsg) The Gameful world: approaches, issues, applications. MIT Press, Cambridge, London, S597-623

61. Koivisto J, Hamari J (2014) Demographic differences in perceived benefits from gamification. Comput Human Behav 35:179-188. https://doi. org/10.1016/j.chb.2014.03.007 